\title{
Development Of GUI Based Programme For Thermal Characterisation And Life Expectancy Of Transformer Using Matlab
}

\author{
J.Mahesh Yadav ${ }^{[1]}$, Dr.A.Srinivasula Reddy ${ }^{[2]}$ \\ ${ }^{[1]}$ Research Scholar, JNT University, Hyderabad, India \\ ${ }^{[2]}$ Prof. \& Principal, Samskruthi Engineering College, Hyderabad, India
}

\begin{abstract}
Transformers represent the largest portion of capital investment in transmission and distribution substations. In addition, power transformer outages have a considerable economic impact on the operation of an electrical network. The most important parameters governing a transformer's life expectancy is the hot-spot temperature (HST) value and loss of life. Here in this paper by using graphical user interface (GUI) programme we are going to find the hot-spot temperature (HST) with and without harmonics, top oil temperature (TOT) with and without harmonics, efficiency and loss of life. This approach has been made to consider the hot-spot temperature as the sum of the ambient temperature, the top-oil temperature rise in tank. With MATLAB-GUI we are finding the [TOT], [HST], Efficiency and loss of life. This paper is applied on a 220KVA, 20KV, ONAF type transformer.
\end{abstract}

Keywords: Efficiency, Harmonics effects, , Hotspot temperature, Loss of life, MATLAB GUI, Transformer, Top oil temperature.

\section{Introduction:}

Transformer is one of the important components of power system, because of harmonics and short circuits occurring in network will increase the temperature rise in windings. Hotspot regions of inordinate temperature rise in the windings and top oil temperature causes the rapid thermal degradation of insulation and thermal run away [1]. It is essential to predict the thermal behaviours of a transformer during normal cyclic loadings and particularly in the presence of overload conditions or unplanned conditions or unplanned outages, further therefore improved knowledge of transformer thermal characteristics can allow the enhanced circuit ratings and reduces the risk [2]. Generally there are many methods to predict the TOT, HST, Efficiency and loss of life [3][4]. However the conventional calculations are done using simulations and most of it is not available for the end user. Here in this paper increased transformer temperature due to time varying loads and ambient temperatures is proposed.In this paper we are adopting a MATLAB-GUI based approach so that a technical layman can use this Graphical user interface [GUI] to get the required output. The developed GUI is user friendly, it also makes it easier to adjust parameters and visualize your programs. i.e., any transformer designers can use this GUI without having software background and need not solve differential equations. Here we have developed a MATLAB GUI based interface for top oil rise temperature with and without harmonics, hottest spot temperature with and without harmonics, efficiency of a transformer and loss of life under various conditions.

In this we have used a simple input output style layout for the GUI and various inputs are taken from edit text boxes and the outputs are delivered to required text box. Most of the non-variable inputs are kept in the MATLAB program itself and most important ones are kept on the GUI. The trigger for the program execution is a push button, when pressed it executes the program behind the interface and results are obtained. In case the results are in the form of graphs, suitable plots are obtained in the layout itself.

\section{Development Of Gui Based Approach:}

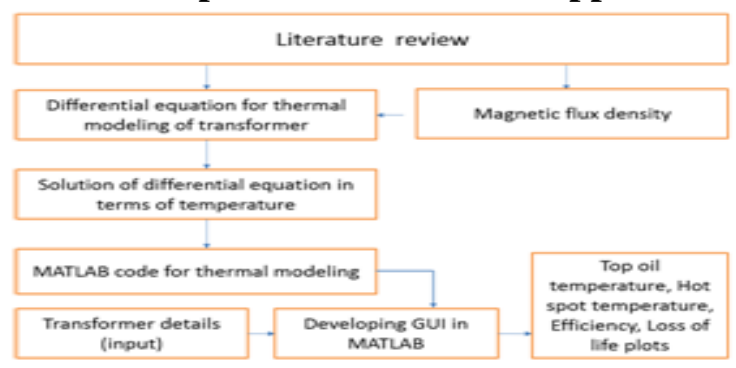

Fig: 1: block diagram of GUI development 
3.1 Ambient temperature

\section{Determination Of The Inputs:}

\subsection{Impact of harmonics 3.3 Other input parameters}

\subsection{Effect of Ambient Temperatures on Transformer:}

Ambient temperature simply means "the temperature of the surroundings" and it is the most important criterion to determine the hottest spot of a transformer. The sum of ambient temperature and increase in top oil temperature gives final top oil temperature.

The insulation hot spot temperature (HST) represents the most important limiting factor of a transformer insulation life. Ambient temperature involved in the HST computations in all transformer thermal models affects insulation life-time directly or indirectly. Regarding the importance of the ambient temperature in transformer's insulation life, the effect of the ambient temperature on HST and transformer insulation life is calculated. Temperature ratings are based on a 24 hours ambient of $30^{\circ} \mathrm{C}$. This is ambient used in guide. Whenever the actual ambient can be measured, such ambient should be averaged over $24 \mathrm{~h}$ and then used in determining the transformer's temperature and loading capability [5].

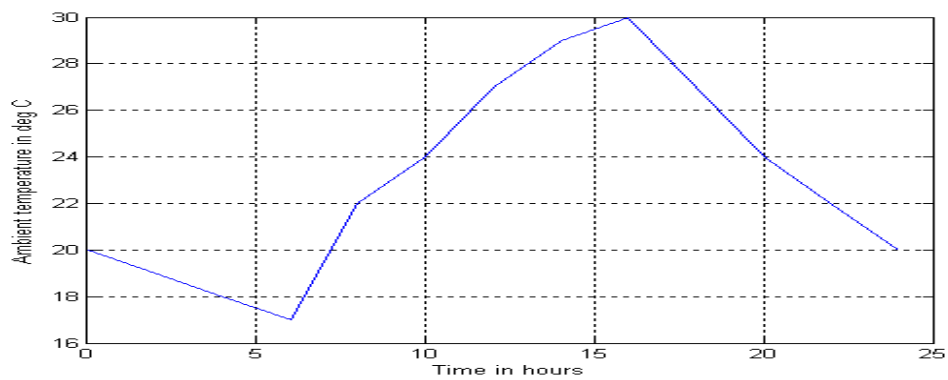

\subsection{Effect of harmonics on Transformer:}

Fig 2: ambient temperature

The use of non-linear (solid state) loads in power system has increased the awareness of the potential reduction of a transformer life due to the increased losses. Manufacturers and users have been applying IEEE C57.110-1989 guidelines which gives a conservative estimate of loading capability of transformers. In this paper, the effect of harmonics on transformers is discussed to predict a rise in top oil, hot spot temperature, efficiency and loss of life. The model considers a time varying harmonic load cycle and ambient temperature.

Current harmonics have most degrading effect on the machine. The harmonic currents will create great amount of stress on insulations and cause the life of equipment to decrease rapidly. Additional losses will be created due to harmonic current components in the windings and other structural parts. Transformers losses $\left(\mathrm{P}_{\mathrm{T}}\right)$ are divided in to no load losses $\left(\mathrm{P}_{\mathrm{NL}}\right)$ and load losses $\left(\mathrm{P}_{\mathrm{LL}}\right)$. No load losses are due to the voltage excitation of the core and load losses will occur in winding of the transformer and it is expressed as:

$$
\mathbf{P}_{\mathrm{LL}}=\mathbf{I}^{2} \mathbf{R}+\mathbf{P}_{\mathrm{EC}}+\mathbf{P}_{\mathrm{OSL}}
$$

The difference between Total loses and $I^{2} \mathrm{R}$ losses is equal to the stray losses. The total losses Measured during the impedance test and there is no test method to distinguish eddy losses from the stray losses that occur in structural parts [5]. Eddy current losses in windings are generated by the electromagnetic flux and they vary with the square of the rms current and the square of the frequency (harmonic order $h$ ) as

$$
\mathbf{P}_{\mathrm{EC}}=\mathbf{P}_{\mathrm{EC}-\mathrm{R}}=\sum_{h=1}^{h=\max } \mathrm{h}^{2}\left(\mathbf{I}_{\mathbf{h}} / \mathbf{I}_{\mathbf{R}}\right)^{2}
$$

The flux magnitude is proportional to the voltage harmonic and inversely proportional to the harmonic order. The harmonic distortion of the system voltage usually 5\% in power systems [6][7]. Therefore neglecting the effect of voltage harmonics and considering $\mathrm{P}_{\mathrm{NL}}$ by fundamental components only.

\subsection{Input Parameters:}

The following is the data table of input parameters

\begin{tabular}{|ll|}
\hline Input Variables & Value \\
Top oil temperature & \\
At rated load $(b)$ & 36 \\
Time difference $\left(\mathbf{d}_{\mathbf{t}}\right)$ & 20 \\
No load losses $\mathbf{P}_{\mathrm{NL}}$ & 153 \\
Initial top oil temperature $\left(\mathbf{T}_{\left.\text {oil_i}_{i}\right)}\right.$ & 39 \\
Hotspot at rated load $(b 1)$ & 55 \\
\hline
\end{tabular}




\section{Top Oil}

Temperature with and without harmonics.

The internal flow of heat from the parts in which it is actually generated (windings) to the cooling medium at initial conditions is called thermal cycle. This heat must be conducted out of the windings and core and dissipated to the surrounding, or ambient air. There's a time delay involved in getting this heat out; as a result, the transformer temperature increases. The difference in temperature between a non-operating transformer and one at full load equilibrium point is called temperature rise.

Equations given below are used to estimating top oil temperature without harmonics. And the same equation is used for estimating the top oil temperature with harmonics by updating increased load losses $\left(\mathrm{P}_{\mathrm{LL}-\mathrm{H}}\right)$ value to include harmonic effect [9].

Formula for increased load losses without harmonic

$$
P_{L L-H}=p \cdot \sum\left(\frac{I_{h}}{I_{R}}\right)^{2}
$$

Top oil temperature $\mathrm{b}$ without harmonic is

$$
b=\frac{\left[T_{a m b} \Delta t+\left[\frac{P_{L L H}+P_{N L}}{P_{L L R}+P_{M L}}\right] * \theta_{f * \Delta t}+\left[\frac{P_{L L R}}{P_{\mathrm{NL}}}\right] *\right. \text { Topoiltemp.(k-1)] }}{\frac{P_{L L R}+\Delta t}{P_{N L}}}
$$

Top oil temperature $b$ with harmonic will be calculated by substituting $\mathrm{P}_{\mathrm{LL}-\mathrm{H}}$ value from equation (3) in the equation (2).

Formula for increased load losses with harmonics

$$
P_{L L-H}=P \cdot \sum\left(\frac{I_{h}}{I_{R}}\right)^{2}+P_{E C} \sum h^{2}\left(\frac{I_{h}}{I_{R}}\right)^{2}+P_{O S L} \sum h^{0.8}\left(\frac{I_{h}}{I_{R}}\right)^{2}
$$

By using the above equations and input variables we designed a simple GUI to calculate the top oil temperature plot. When we run the programme, a graphical interface pop's out. GUI layout which is given below.

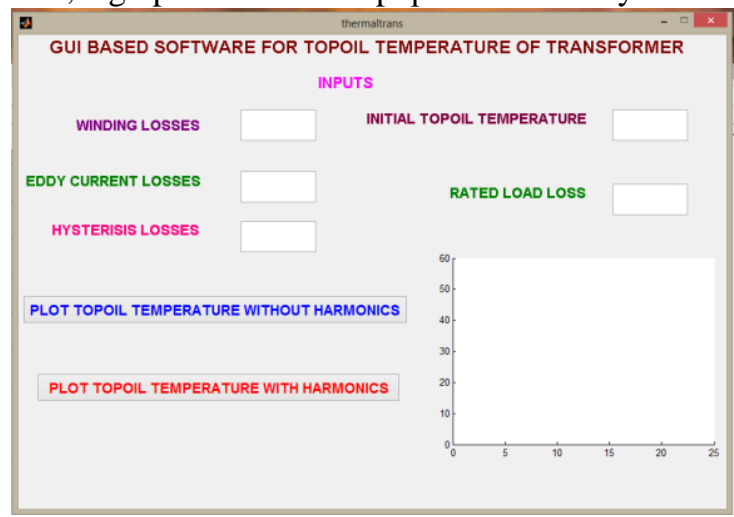

Fig 3: Top Oil Temperature GUI.

The values which we have used for (TOT) calculations is given below,

\begin{tabular}{|ll|}
\hline Input Variables & Value \\
Winding losses & \\
Eddy current loss & 112800 \\
Hysteresis losses & 46 \\
Initial top oil temperature & 98 \\
Rated load loss & 39 \\
\hline
\end{tabular}

After giving inputs when we hit the push button it initialises the execution of the program under the interface and delivers the results to the corresponding destinations. In this the output destination is a plot function so that graph is displayed in the graph plane.

The inputs are taken from edit text windows and "get "function is used to deliver the input values to the programme."str2double" takes consideration of the decimal digits without rounding them to nearest integers. All the green lines of the .m file are auto generated and they will not effect our programme, these codes are required to save the layout of GUI for the next time we run it. Hence we can neglect them in the total programme.

functionL_wind_Callback(hObject, eventdata, handles) 
L_wind_a=str2double(get(handles.L_wind,'string'))

$\%$ hObject handle to L_wind (see GCBO)

$\%$ eventdata reserved - to be defined in a future version of MATLAB

$\%$ handles structure with handles and user data (see GUIDATA)

$\%$ Hints: get(hObject,'String') returns contents of L_wind as text

$\% \quad$ str2double(get(hObject,'String')) returns contents of L_wind as a double

When we hit the push button execution starts at once. But before that we need to specify what the push button needs to do and all the inputs must be declared once again to the programme under push button. Declaring the inputs again in the following lines,

$\%$--- Executes on button press in topoil.

functiontopoil_Callback(hObject, eventdata, handles)

L_hyst_a=str2double(get(handles.L_hyst,'string'))

L_eddy_a=str2double(get(handles.L_eddy,'string'))

L_wind_a $=$ str2double(get(handles.L_wind,'string'))

P_LL_R_a=str2double(get(handles.P_LL_R,'string'))

$\mathrm{dt}=20$;

$\mathrm{b}=36$;

c=P_LL_R_a/Loss_noload;

then calculating the total top oil temperature in the system considering all the variables below,

for $k=2: 13$

$a=\left(\left(P \_L L \_H(k, 1)+L o s s \_n o l o a d\right) /\left(P \_L L \_R \_a+L o s s \_n o l o a d\right)\right)$;

Temp_topoil $(k, 1)=\left(\left(\left(T e m p \_a m b(k, 1) * d t\right)+a * b * d t+c * T e m p \_t o p o i l(k-1,1)\right) /(c+d t)\right)$

After the result is obtained it is saved in Temp_Topoil matrix then by plotting this to a graph divided by two hours interval for a total of 24 hours of a day load cycle the code is shown below.Plot function is used to send the results directly to the graph plane,

time=0:2:24;

$\%$

plot(time,hotspot, 'b','LineWidth',3)

ylabel('top oil temperature in deg $C^{\prime}$, 'fontsize',18)

xlabel('Time in hours','fontsize',18)

gridon

holdon

Time is taken in two hours interval for 24 hours.

Plotting time vs top oil temperature

and labelling $\mathrm{x}$ and $\mathrm{y}$ axis with suitable names.

Grid on for a grid lines that makes the graph easier to read.

Hold on for holds the current plot and all axis properties so that subsequent graphing commands add to the existing graph.

After executing the GUI (TOT) the results obtained are given below.

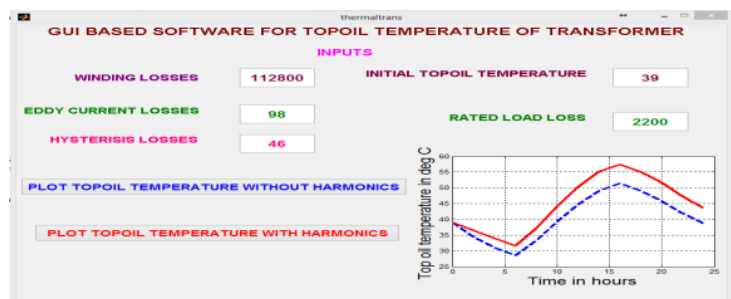

With harmonics

without harmonics

Fig 4: Results in GUI of (TOT) Interface. 


\section{Hottest Spot With And Without Harmonics:}

The temperature rise is averaged over the whole winding; the inside of a winding is hotter than it's outside, in reality. The hottest spot is at some point inside the coil having the longest thermal paths to the outside air. It's usually expressed as a temperature increase over the average temperature. The temperature rise must be limited by design and application so that the total temperature does not exceed the temperature rating of the insulation used.

The equation (4) given below is used to estimate HST without harmonics and equation (1) is used to calculate $\mathrm{P}_{\text {LLH }}$.

$$
T_{H}=\left[\frac{T_{\text {oil }} \cdot \Delta t+\left[\frac{\mathrm{P}_{\mathrm{LLH}}+\mathrm{P}_{\mathrm{NL}}}{P_{L L R}+P_{\mathrm{NL}}}\right] \cdot b 1 \Delta t+c T_{H} \cdot(k-1)}{c+\Delta t}\right]
$$

By substituting the value of $\mathrm{P}_{\mathrm{LLH}}$ from equation (3) in equation (4) we will get HST with harmonics. By using equations (3) and (4) we get a GUI of HST. The corresponding interface is given below.

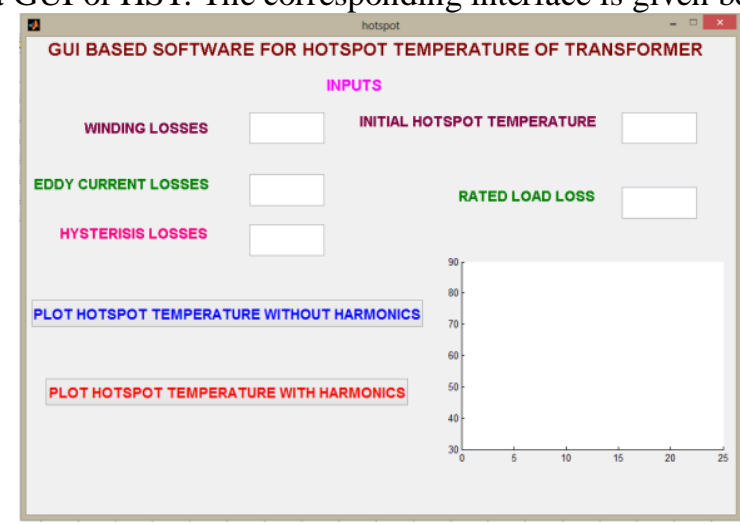

Fig: 5: Hottest spot Temperature GUI.

The input variables to calculate HST are,

\begin{tabular}{|lc|}
\hline Input Variables & Value \\
Transformer rating & $\mathbf{2 2 0}$ \\
Frequency & 50 \\
Rated current & 10 \\
Eddy constant & 30 \\
Hysteresis constant & 30 \\
Magnetic flux density & 1.1 \\
\hline
\end{tabular}

After executing the GUI (HOT) the results obtained are given below.

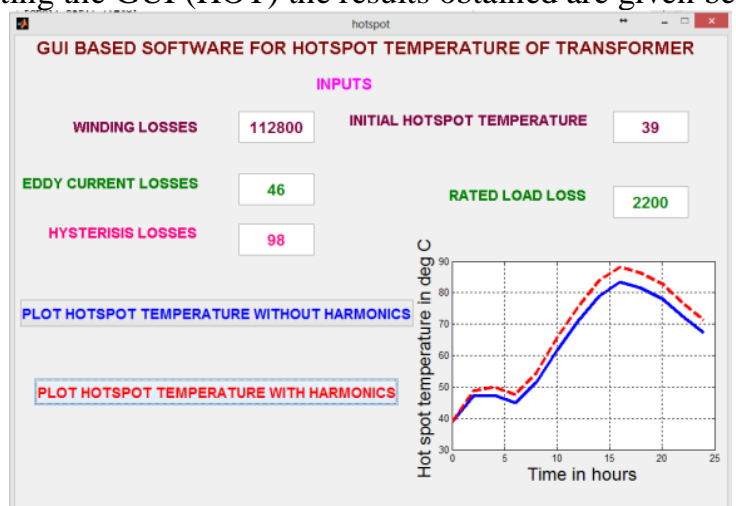

Without harmonics

with harmonics

Fig: 6: Results of Hotspot Temperature with and without harmonics using GUI. 


\section{Estimation Of Efficiency:}

There are various factors that reduce the efficiency of a transformer such as eddy current losses, hysteresis losses and leakage of flux. The formula for efficiency is obtained as [10].

$$
\mathbf{y}=1-\frac{\left(P_{N L}+P_{L L}\right)}{S X \cos \theta} X 100
$$

By using the above equation efficiency of the transformer is obtained,

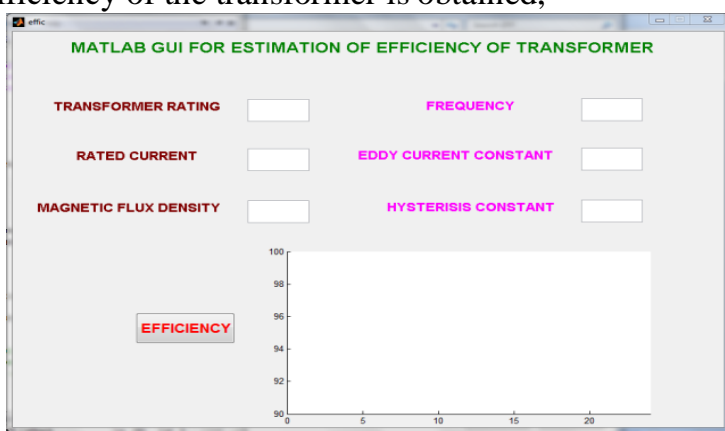

Fig: 7: Estimation of Efficiency using GUI.

The input variables to calculate efficiency,

\begin{tabular}{|ll|}
\hline Input Variables & Value \\
& \\
Winding losses & $\mathbf{1 2 8 0 0}$ \\
Eddy current losses & $\mathbf{4 6}$ \\
Hysteresis losses & $\mathbf{9 8}$ \\
Initial hotspot temperature & $\mathbf{5 1 . 5}$ \\
Transformer rating & $\mathbf{2 2 0}$ \\
\hline
\end{tabular}

After executing the GUI, Efficiency ( $\mathrm{y}$ ) the results obtained as given below.

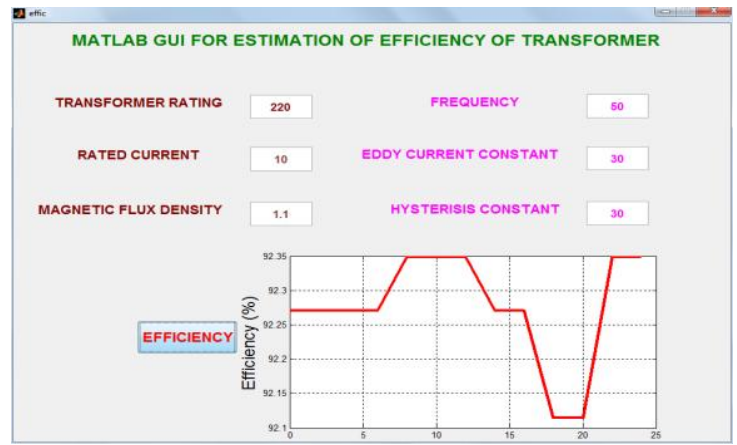

Fig: 8: Estimation of Efficiency results using GUI.

The efficiency mainly depends on the load current, pf and losses.

\section{Loss of Life:}

The Matlab program proceeds through a series of calculations to convert the transformer characteristics to constants which are used in determining the transformer winding hot-spot temperature for each of the 2 hours intervals of the daily load cycle. These hot-spot temperatures are then used by the program to calculate the percent loss of life for each 2 hours interval using equation. The per-cent loss of life per day is the summation of the per-cent loss of life for each 2 hours interval of the daily load cycle [11].

Loss of life $=\frac{0.0000285 \text { xaging } \text { factor } \times 100 \%}{\text { base life }}$

Where $0.0000285=\frac{1}{8790 \times 4}=$ years per quarter hour.

By using the above equation loss of life of the transformer is obtained, 


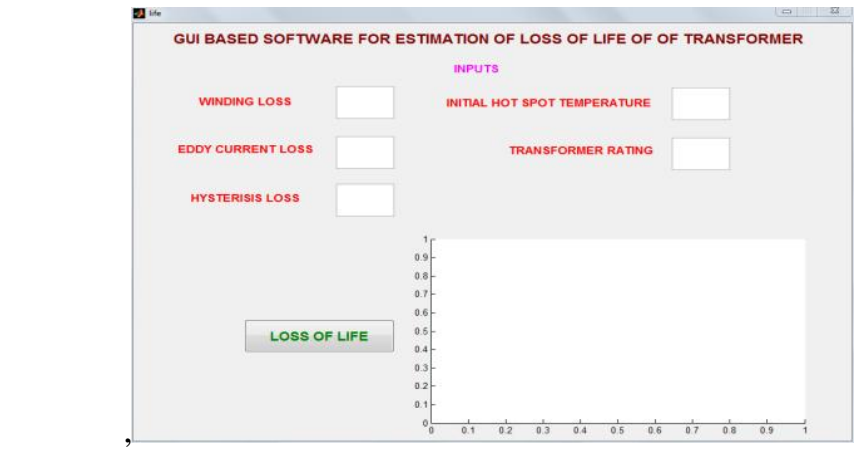

Fig: 9: $\quad$ Estimation of Loss of Life using GUI.

The input variables to calculate loss of life are

After executing the GUI,

\begin{tabular}{|ll|}
\hline Input Variables & Value \\
Winding losses & \\
Eddy current loss & 112800 \\
Hysteresis losses & 46 \\
Initial top oil temperature & 98 \\
Rated load loss & 39 \\
\hline
\end{tabular}

Efficiency $(\mathrm{y})$ the results obtained is given below.

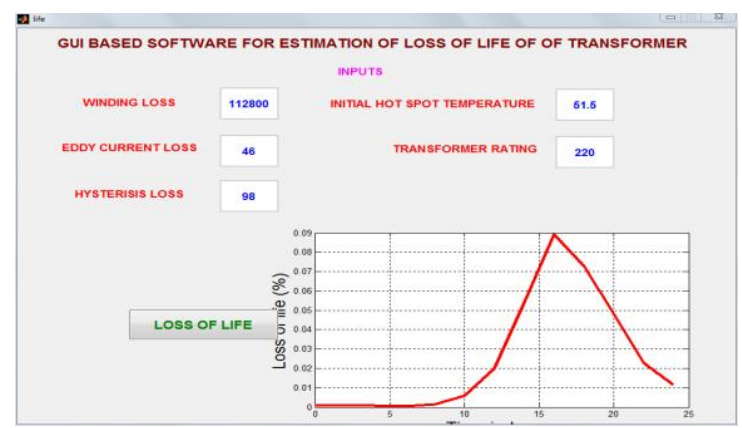

Fig: 10: Estimation of Loss of Life results using GUI.

\section{Conclusion:}

Top oil temperature, hottest spot temperature, Efficiency and Loss of life are calculated with and without harmonics for a transformer using GUI. This approach is simple yet powerful to follow and any technical layman can understand it. Its approach is simple as the most essential input parameters are kept on the GUI and the remaining are declared in the programme itself to avoid confusion. This package can be used by the transformer manufactures to predict the thermal behaviour of the transformer before even designing it. . The top oil temperature calculated from the top oil equation becomes the ambient temperature for hot spot equation model. To correctly estimate a transformer loss of life the real load and ambient temperature variations should be considered.

\section{References:}

[1]. IEEE std c57-110-1998, recommended practice for Establishing Transformer capability when supplying non sinusoidal load

[2]. "Simple Thermal Model of Transformer Hot Spot Temperature Based on Thermal-Electrical Analogy ." Xiaoping Su1, WeigenChen , Chong Pan, Qu Zhou, Li Teng.

[3]. IEEE guide to loading mineral oil immersed power transformers, international electrochemical commission standards geneve, Suisse 1991.

[4]. IEEE standard C57.91-1995(R2004) IEEE guide for mineral oil immersed transformers.

[5]. IEEE std C57.91-1995(R2004) "IEEE guide for loading mineral oil immersed transformer."

[6]. A.Elmoudi, M.Lehtonen, HasseNordman, conference record of the 2006 IEEE International symposium on Electrical Insulation. "Effect of Harmonics on Transformer Loss of Life.

[7]. M.T.Bishop, J.F.Barnowski, D.Heath, S.J.Benna, "Evaluating harmonics induced transformer heating." IEEE trans. on power delivery, vol.11, No 1, Jan 1996, pp.305-311.

[8]. A. Girgis, E.Makram, and J Nims, "Evaluation of Temperature Rise of Distribution Transformers in the presence of Harmonics and distortions." Electrical Power Systems Research, Vol 20, No 1, Jan 1990. pp.15-22. 
[9]. G. Swift, T. S. Moliniski, and W. Lehn, "A Fundamental Approach to Transformer Thermal Modeling-Part I: Theory and Equivalent Circuit,"IEEE Trans. Power Delivery, vol. 16, No.2, Apr. 2001, pp. 171-175.Robert E.Rood "A method for estimating the thermal expectancy of distribution transformer." Approved by the AIEE presented at the IEEE Winter general meeting.

[10]. KshiraT.Muthanna, Abhinanda Sarkar, Kaushik Das, and Kurt Waldner, IEEE tans.power delivery,vol.21,NO.1,Jan 2006."Transformer Insulation Life Assessment."

\section{Nomenclature:}

$\begin{array}{lll}\mathrm{I}_{\mathrm{h}} & - & \text { Harmonic load current at different intervals } \\ \mathrm{P} & - & \mathrm{I}^{2} \mathrm{R} \text { Winding losses } \\ \mathrm{P}_{\mathrm{EC}} & - & \text { Eddy current losses } \\ \mathrm{K}_{\mathrm{e}} & - & \text { Eddy current loss factor } \\ \mathrm{f} & - & \text { Frequency } \\ \mathrm{B} & - & \text { Magnetic flux density } \\ \mathrm{t} & - & \text { Time } \\ \mathrm{V} & - & \text { Voltage that corresponds to eddy current loss } \\ \mathrm{P}_{\mathrm{OSL}} & - & \text { Stray losses. } \\ \mathrm{K}_{\mathrm{f}} & - & \text { Hysteresis loss factor } \\ \mathrm{P}_{\mathrm{LL}-\mathrm{H}} & - & \text { Increased load losses } \\ \mathrm{I} & - & \text { Rated current } \\ \mathrm{d}_{\mathrm{t}} & - & \text { Time difference } \\ \mathrm{P}_{\mathrm{NL}} & - & \text { No load losses } \\ \mathrm{P}_{\mathrm{LL}-\mathrm{R}} & - & \text { Rated load losses } \\ \mathrm{V} & - & \text { Rated voltage } \\ \mathrm{b} & - & \text { Top oil temperature at rated load } \\ \mathrm{b} 1 & - & \text { Hottest spot temperature at rated load } \\ \mathrm{c} & - & \text { ( P } \\ \mathrm{T}_{\text {oil_initial }} \text { - } / \text { P } & \text { Initial top oil temperature } \\ \text { app } & \text { - } & \text { Apparent power } \\ \mathrm{y} & - & \text { Efficiency } \\ \end{array}$

